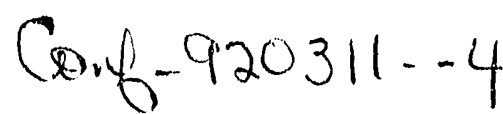

Page 1

\section{Noble Gas Pumping by the TFTR Graphite Limiter}

\author{
A. T. Ramsey and D. M. Manos \\ Princeton Plasma Physics Laboratory \\ Princeton University \\ Princeton, NJ 08543 U.S.A.
}

PPPL-CFP-2605

DE92 012669

\title{
Abstract
}

The TFTR limiter is graphite. It is possible to decrease the amount of D trapped in the limiter by making plasma discharges with gas injected only as a prefill. When the limiter is deuterium depleted well enough by this technique for supershots (with $R_{D} \sim 0.5-0.6$ ) it pumps noble gases. The effective confinement time $\tau_{\mathrm{p}}{ }^{*}$ ranges from $\leq 1 \mathrm{~s}$ for He down to $0.08 \mathrm{~s}$ for $\mathrm{Kr}$. These noble gases escape from the limiter slowly after the run. The partial pressure of Ar and Ne decay with time-constants of several hours, and the data for Ar show evidence of two trapping sites.

\section{Introduction}

The limiter of TFTR is a graphite wall, which covers the inner side of the torus from $60^{\circ}$ to $+60^{\circ}$, measured from the midplane. It has an area of $22 \mathrm{~m}^{2}$ and a mass of $2000 \mathrm{~kg}$. During operation in low-mode, it recycles deuterium with $\mathrm{R}_{\mathrm{D}} \leq 1$. Strachan and coworkers discovered that the limiter recycling could be sharply reduced by running a sequence of conditioning shots (15-30) with only enough gas puffed into the torus before the shot to allow electrical breakdown and plasma formation. It was this conditioning that led to their discovery of the TFTR enhanced (by 2 to 3 over low-mode) energy confinement discharges we call "supershots."[1] The conditioning shots are usually pre-filled with He, although toward the end of a conditioning sequence $Z_{\text {eff }}$ will approach 6 , indicating that the limiter is being scoured primarily by carbon ions. In the absence of external fuelling sources, the recycling coefficient $R$ is defined by the relation

$$
\frac{\delta N_{p}}{\delta t}=-\frac{N_{p}}{\tau_{p}}+R \frac{N_{p}}{\tau_{p}}
$$

where $\tau_{p}$ is the global particle confinement time of the plasma, and $N_{p}$ is the number of particles of the type observed. We can define an effective particle confinement time, $\tau_{\mathrm{p}}{ }^{*}$, as

2)

$$
\tau_{p}^{*}=\frac{\tau_{p}}{(1-R)}
$$

and note that this is what we measure when we watch the decay of the brightness of an influx line or of the density increment after a gas puff.

When conditioning is done, the limiter recycling of $D$ has dropped to $R_{D} \sim 0.5-0.6$, and a puff of $D_{2}$ into an ohmic plasma decays away with a characteristic time of $\tau_{D}^{*}=$ 0.12-0.15 s. A full description of the conditioning process has been given by Dylla.[2]

In this paper we shall show that the graphite limiter pumps noble gases when it is conditioned, and does not pump them when it is saturated with D. In the usual low-mode 


\section{DISCLAIMER}

This report was prepared as an account of work sponsored by an agency of the United States Government. Neither the United States Government nor any agency thereof, nor any of their employees, makes any warranty, express or implied, or assumes any legal liability or responsibility for the accuracy, completeness, or usefulness of any information, apparatus, product, or process disclosed, or represents that its use would not infringe privately owned rights. Reference herein to any specific commercial product, process, or service by trade name, trademark, manufacturer, or otherwise does not necessarily constitute or imply its endorsement, recommendation, or favoring by the United States Government or any agency thereof. The views and opinions of authors expressed herein do not necessarily state or reflect those of the United States Government or any agency thercof. 
case, the noble gases recycle with $\mathrm{R} \sim 1$. Moreover, we shall show that the trapping time in the limiter can be substantial. After a run, we see the noble gas partial pressure declining with characteristic times of several hours.

In Section 2, we shall present data on the plasma-limiter pumping interaction for He, $\mathrm{Ne}, \mathrm{Ar}$, and $\mathrm{Kr}$, and compare it with the pumping of $\mathrm{D}$. We shall argue that the noble gases are either being trapped in the same sites used by $D$ or are blocked by trapped $D$ from reaching trapping sites. In Section 3, we shall show the limiter outgassing of Ar and Ne after a run in which they were used. Argon partial pressures show a clear double decay suggesting the presence of 2 traps, one substantially deeper than the other. Neon decay data support this. Simultaneous $\mathrm{D}_{2}$ partial pressure decay measurements indicate that $\mathrm{Ar}$ and $\mathrm{Ne}$ are sharing trapping sites not used by $\mathrm{D}$. Finally, we shall show data which suggests that the deeper trap is already present in natural graphite and the shallower trap is created by damage in a plasma discharge.

\section{Plasma Pumping}

Figure 1 shows the effect of conditioning on the evolution of the line integrated density from a puff of $\mathrm{He}$ into a neutral beam heated plasma. In both cases, $12 \mathrm{MW}$ of neutral beam power were being injected. The solid curve shows the usual behavior seen in lowmode discharges. The dashed curve shows what happens when ihe limiter has been conditioned so that $R_{D} \sim 0.5$. The same behavior in the unconditioned state is observed in other gases. too. Figure 2 shows the $\mathrm{Z}_{\text {eff }}$ for 3 shots into which $1 \mathrm{~T}-\ell$ of $\mathrm{Ne}$ was puffed into an ohmic conditioning shot between 3.0 and 3.2 seconds. The shots are separated by 7 non-Ar conditioning shots each. The first shot shows unconditioned behavior. The fueling efficiency of this shot is less than the last shot $\left(\Delta \mathrm{Z}_{\mathrm{eff}} \sim 2 v s . \Delta \mathrm{Z}_{\mathrm{eff}} \sim 6\right.$ for the same amount of $\mathrm{Ne}$ ), probably due to changes in the edge density. However, for an unconditioned limiter, the $\mathrm{Z}_{\text {eff }}$ changes after the puff less than $\Delta \mathrm{Z}_{\text {eff }} \sim 0.1$ for the duration of the discharge. The fueling efficiency improves as the conditioning progresses, but the $\mathrm{Z}_{\text {eff }}$ drops strongly after the puff, showing limiter pumping. The improving conditioning is seen directly in the pre-Ne puff $\mathrm{Z}_{\text {eff }}$ as it climbs from $\mathrm{Z}_{\text {eff }} \sim 1.8$ to $\mathrm{Z}_{\text {eff }} \sim 3.816$ shots later. As the $D$ effusion from the limiter falls, the edge temperature rises, and the $C$ concentration (largely from self-sputtering) increases sharply.

We can measure the rate of pumpout of the Ne by looking at the decaying brightness of an influx line (such as Ne VII at $465 \AA$ ), or the fall of the density. For a conditioned limiter, both techniques give the same answer. Figure 3 shows the density decay for the last (highest $Z_{\text {eff }}$ ) shot in Fig. 2. The decay time of $\tau_{\mathrm{Ne}}{ }^{*}=0.7 \mathrm{~s}$ for this shot can be compared to an energy confinement time of $\tau_{\mathrm{E}} \sim 0.15 \mathrm{~s}$ and a particle confinement time of $\tau_{p} \sim 0.2-0.3 \mathrm{~s}$ (from an $\mathrm{H}_{\alpha}$-based fueling calculation), and indicates a recycling coefficient of $R_{\mathrm{Ne}} \sim 0.6$. (We are assuming here that $\tau_{\mathrm{p}}(\mathrm{Ne})=\tau_{\mathrm{p}}(\mathrm{D})$, which is our observation on TFTR in most operating regimes.)

Figure 4 shows the brightness of the Ar XVI line at $354 \AA$ for three consecutive 12 MW supershots into which 0.2, 0.5, and 2.3 T- $\ell$ of Ar were puffed. Even though the Ar pumpout between shots is substantial there is clear evidence of some buildup of the prepuff Ar levels. A fit to the brightest shot gives a characteristic time of $\tau_{\mathrm{Ar}}{ }^{*}=0.18 \pm 0.02$ s. 
For He puffing, the behavior is much the same. In both ohmic and beam heated shots, the pumpout of the He is about $0.9 \mathrm{~s}$, as shown in Fig. 1 (for a plasma current of 1.4 MA). Table 1 summarizes the effective confinement times for the noble gases and deuterium. The pumpout behavior of $\mathrm{Kr}$ puffs are similar to those shown above, and only the results are shown in the table.

To summarize, we can say that the pumpout of the noble gases tracks the pumpout of deuterium. The degree of pumpout (although not the rate, as far as we can tell) depends on the condition of the limiter. Heavier gases pump out faster (with $\mathrm{Kr}$ even faster than $\mathrm{D}$ ). The effect is the same in ohmic and beam heated plasmas, and is connected to the plasma confinement and limiter recycling, not the heating method. It seems most likely that the noble gases either compete with D for the same bulk trapping sites in the graphite or that the deuterons physically obstruct access to alternative suitable sites on the lattice. As D is desorbed through carbon ion bombardment in the conditioning shots, sites become available for the noble gases.

\section{Limiter Outgassing}

We have measured the partial pressure of Ar and Ne during the 7 hours between tixe end of plasma operations at midnight and startup the next morning. For pressures well above the blank-off pressure of the turbopumps, the relation between the outgassing rate, $\mathrm{Q}$, the partial pressure, $\mathrm{p}$, and tine vessel's pumping speed $\mathrm{S}$ and volume $\mathrm{V}$ is

$$
\mathrm{Q}=\frac{\delta \mathrm{p}}{\delta \mathrm{t}} \mathrm{V}+\mathrm{Sp}
$$

The term involving $\delta p / \delta t$ is less than $1 \%$ of the term in $p$ for the cases considered here, so we may take the pressure as being directly proportional to the outgassing. Our best data is from the Ar puffing experiments; the Ne partial pressures were lower and noisier. (The partial pressure of $\mathrm{He}$ cannot be distinguished from $\mathrm{D}_{2}$, and $\mathrm{Kr}$ falls outside our instrument's measurement range.) All data were taken by the TFTR residual gas analyzer system. Figure 5 shows the partial pressure of ${ }^{40} \mathrm{Ar}$ following the run which produced the data in Fig. 4. The data are fit with a single exponential curve on a pedestal, and the residuals of the fit (in percent of the pressure) are shown below. An exponential decay is characteristic of first-order release of the gas atoms from traps. Inverse power law decay with time (of the form $\mathrm{P}=\mathrm{P}_{0} \mathrm{t}^{-\mathrm{b}}$, where $\mathrm{b}$ falls between 0.5 and 1.0 ) is associated with diffusive release from various materials.[3][4] We propose that the observed first-order loss of $\mathrm{Ar}$ (and $\mathrm{Ne}$ ) from the graphite limiter is controlled by detrapping, followed by subsequent rapid transport to the surface and desorption.

While the process is clearly not a power law, the residuals in Fig. 5 show that the parent distribution of the data is alsn not a purc exponential. The outgassing rate should have a strong dependence on the limiter temperature, and we know that the surface of the limiter becomes hot during plasma discharges. At raised points, which intercept an anomalous amount of power, the temperature has reached $2300 \mathrm{~K}$.[5] The average is much lower, and the bulk temperature is moderate, reaching perhaps $360 \mathrm{~K}$ during a shot, and cooling with a time constant of about 15 minutes. At the end of a day's operations, the limiter cooling is left on until the temperature is about $300 \mathrm{~K}$. This temperature remains 
more or less constant overnight. Therefore, on the time scale of these experiments, the temperature may be regarded as constant and we may look for another explanation.

We fit the data in Fig. 5 with an unconstrained double exponential, shown in Figure 6. We see that $34 \%$ of the Ar decays with a time constant of $\tau_{\mathrm{Ar}}$ (short) $=0.87 \pm 0.05$ hours, and $66 \%$ decays with $\tau_{\mathrm{Ar}}(\mathrm{long})=4.1 \pm 0.3$ hours. We inadvertently performed an experiment which supports this two time constant interpretation of the outgassing. We ran a magnetron sputtering discharge in Ar in TFTR to boronize the interior of the vacuum vessel. There was a large amount of Ar co-deposited or buried in the limiter in this process, but the geometry of the sputtering apparatus and the manner of its operation led to little or no ion bombardment of the walls during the deposition. If we examine the outgassing after the run, we find that there is a single time constant, with $\tau_{\mathrm{Ar}}=5.3 \pm 0.1$ hours. These data, shown in Fig. 7, show no strong pattern in the residuals as the data in Fig. 5 do, and a double exponential fit yields a second element which is consistent with a zero amplitude. Immediately after the data in Fig. 7 were taken, we attempted plasma operations. After 28 very poor deuterium discharges (many disrupted) we stopped to bake out the machine to get rid of the argon. Before we did, we were able to record outgassing data for 7 hours. At this time, the shorter time constant had returned, indicating that the plasma operation, brie $i$ as it was, had created the shallow traps.

Finally, we analyzed the Ne partial pressure decay after a Ne puffing run. The pressure was much lower, with the $22 \mathrm{Ne}$ peak decaying from $5 \times 10^{-11}$ Torr to $2 \times 10^{-11}$ Torr in 7 hours (compare with the $1.5 \times 10^{-9}$ Torr of the $40 \mathrm{Ar}$ in Figs. 4 and 5). While the noise is greater, an unconstrained double exponential fit finds the same fraction in short and long lived traps as for the Ar data of Fig. 5 .

The outgassing data are summarized in Table 2 . The problem of errors in multiparameter non-linear least squares fitting is always a vexing one. We have quoted the fitting errors given by a commercial software package, Kaleidagraph.[6] The variables are not orthogonal, and hence the quoted errors probably should be regarded as a lower bound. Table 2 also shows $\mathrm{D}_{2}$ outgassing after the Ar glow and subsequent series of plasma discharges. It, too, has a double time constant, but the relative population of the two sites is quite different from Ar. This suggests that the two species are not using the same traps.

\section{Discussion}

There is more than one way in which gases may be incorporated into the graphite in TFTR. Gases may be physically incorporated into the "co-deposition layer" which is formed when the carbon sputtered in the zones of high ion flux is redeposited on the limiter.[7] Gases may also be implanted into the graphite as impinging plasma ions. The incorporation of inert gases, other than $\mathrm{He}$, into deposited semiconductor and metallic thin films has been the subject of previous studies. [8] However, similar studies for carbon films have not specifically addressed this issue [9], but have concentrated on the lattice bond changes associated with ion bombardment. Numerous studies of He trapping and release, and deuterium trapping and release with and without the mediating effects of $\mathrm{He}$ ion bombardment have been performed.[10][11][12][13][14][15] A common feature of these studies is that damage associated with prior He ion bombardment (at energies from 8 $\mathrm{keV}$ to $100 \mathrm{keV}$ ) led to increased hydrogen trap binding energies, as well as trap formation decper in the material.[14][15] The thermal desorption studies of Atsumi indicate that 
initially He occupies interstitial sites of high mobility, but that progressive bombardment permits the formation of vacancy clusters or interstitial dislocation loops having higher binding energy ( $0.9 \mathrm{eV}$ up from $0.7 \mathrm{eV}$ at low fluence) and greater concentration of He.[11] This second type of trap appears to saturate at a fluence of approximately $5 \times 10^{17} \mathrm{~cm}^{-2}$, depending somewhat on the impinging energy. The amount of retained $\mathrm{He}$ in those studies varied from $12 \%$ to $22 \%$ depending on the original morphology of the samples. These activation energies are far below the transgranular diffusion activation energies for hydrogen and deuterium $(2.8 \mathrm{eV})$. Nevertheless they are still large enough that at the low limiter temperatures in the present studies no interstitial diffusion would be expected for He. Permeability studies of $\mathrm{He}, \mathrm{Ne}$, and Ar performed on graphite HTGR reactor materials by Powell[16] give transgranular diffusion coefficients of $D_{0} \mathrm{e}^{-1.2(\mathrm{eV}) / \mathrm{kT}}$ for $\mathrm{He}, \mathrm{D}_{0} \mathrm{e}^{-1.8(\mathrm{eV}) / \mathrm{kT}}$ for $\mathrm{Ne}$, and $\mathrm{D}_{0} \mathrm{e}^{-2.8(\mathrm{eV}) / \mathrm{kT}}$ for $\mathrm{Ar}$, where $\mathrm{D}_{0}$ is $1 \times 10^{-6} \mathrm{~m}^{2} \mathrm{~s}^{-1}$ for all three gases, and the available volume of the pores was approximately $4.6 \%$ for all gases. Again this predicts that pore diffusion is entirely negligible for the temperatures in this study.

Pore diffusion of deuterium in graphite is known to have a very much lower activation energy $(0.9 \mathrm{eV})$, associated with the weaker chemisorption of deuterium atoms in C-D and $\mathrm{C}_{2}-\mathrm{D}$ bords. It is reasonable to expect that dangling bonds and damage sites on the interior of such pores, if not occupied by deuterium, would be available for still weaker chemi- or physisorption of inert gas atoms. Halsey has performed BET studies of glasses and graphites, from which he was able to extract van der Waals interaction energies for the adsorption of inerts (except $\mathrm{He}$ ) on graphite.[17] His observed values are as follows: 435 $\mathrm{K}$. for $\mathrm{Ne}, 1229 \mathrm{~K}$ for $\mathrm{Ar}, 1651 \mathrm{~K}$ for $\mathrm{Kr}$, and $2117 \mathrm{~K}$ for $\mathrm{Xe}$. If we assume that the rate limiting step for the release in our studies is the desorption of inerts from interior surfaces of pores which are well-connected to the surface, and that the desorption step at a fixed temperature will ha. ve a first-order Arrhenius rate with an activation energy equal to the above values, then the qualitative ordering of our observed release times can be explained. Quantitatively, however, one would predict that the rate coefficient for release of Ne would be 14 times larger than that for Ar, and since the occluded volumes of these materials on graphite arc comparable, the resilting decay times should be in the same proportion. The existence of traps associated with ion bombardment, analogous to the case for hydrogen, and the possibility of analogous saturable damage accumulation sites would complicate the simple picture considerably however. Based on these considerations we propose that the majority of the inert gas trapping in these experiments was ass $y$ ciated with incorporation in a codeposited layer where it chemisorbed on the surface of interior pores, which are relatively well connected to the surface.

Ion bombardment leads to the formation of additional trapping sites in the near surface layer. The range for $800 \mathrm{eV}$ He in graphite is $80 \AA$, the range for $8 \mathrm{keV}$ Ar in graphite is $100 \AA$; these energies represent a typical impinging charge state of each ion through a sheath in a mostly deuterium plasma having $\mathrm{T}_{\mathrm{e}}$ of $100 \mathrm{eV}$. The codeposited layer in TFTR grows at a typical rate of 20 to $40 \AA$ per discharge. The formation of the damage induced trap', will occur throughout the depositing layer and well into the underlying layer from previcus discharges, although the majority of damage will still be caused by deuteriım and carbon bombardment since the inert gas flux is only a brief perturbation to the fuelling of the discharge and carbon sputtering release will be continuously occuring. 
The outgassing data in Table 2 above suggests that the number of traps increases by approximately $50 \%$ during ion bombardment, but unlike the case for hydrogen, the additional traps resulting from bombardment are energetically shallower than those associated with the pore surfaces in the co-deposited layer.

Our observations for inert gases in graphite also contrast with the results for trapping of inert gases in $\mathrm{W}$ reported by Kornelsen, who reported that the activation energies for the release of all inerts were independent of species, suggesting that release was dominated by rearrangements of the W lattice with increasing temperature.[18] $\mathrm{He}$ found that ion bombardment induced an independent group of traps at greater depth in the lattice having increased binding energy ( 1.5 to $4 \mathrm{eV}$ ), the opposite of our observations with Ar in graphite. Details of the nature of the binding for noble gases in carbon must await further laboratory studies which include surface spectroscopies to characterize both the trapped species and the lattice.

\section{Acknowledgements}

The $\mathrm{Kr}$ pumpout data were furnished by $\mathrm{K}$. W. Hill from the $\mathrm{K}_{\alpha}$ lines on his pulse height analyzer system. We thank A. Janos, P. La Marche, and D. K. Owens of PPPL for valuable conversations. D. Walsh and B. Doyle of SNL kindly supplied us with the ranges of He and Ar in C. This work was supported by the United States Department of Energy under Contract Number DE-AC02-76-CHO-3073.

\section{References}

[1] Strachan, J. D., Bitter, M., Ramsey, A. T., Zarnstorff, M. C., Arunasalam, V., et al., Phys. Rev. Lett. 55 (1987) 1004.

[2] Dylla, H. F., Ulrickson, M., Bell, M. G., Owens, D. K., Buchenauer, D., et al., J. Nucl. Matls. 162-164 (1989) 128.

[3] Edwards, E., J. Vac. Sci. Technol. 14 (1977) 606.

[4] Dayton, B. J. in Trans. 8th Nat. Vac. Symp. 1, 42 (1962).

[5] Kamsey, A. T., Bush, C. E., Dylla, H. F., Owens, D. K., Pitcher, C. S., et al., Nuc. Fus. 31 (1991) 1811.

[6] KaleidaGraph, Abelbeck, L., v. 2.1, 1990. Published by Synergy Software, Reading, PA.

[7] Brooks, J. N., Brice, D. K., DeWald, A. E., McGrath, T. T., J. Nuc. Mat. 162 164 (1989) 363.

[8] Desgranges, C., Pellet, C., Schwebel, C., Chabrier, A., Gautherin, G., in Interaction of Charged Particles with Surfaces, Ed. A. Gras-Marti. Pienum Press, 1991. p 675.

[9] Rossnagel, S. M., Russak, M. A., Cuomo, J. J., J. Vac. Sci. Technol. A5 (1987) 2150.

[10] Tokura, S., Atsumi, H., Yamauchi, T., Shinno, M., Yamanaka, S., et al., J. Nucl. Matl. 155-157 (1988) 246.

[11] Atsumi, H., Tokura, S., Yamauchi, T., Yamanaka, S., Miyake, M., J. Nucl. Matl. 141-143 (1988) 258. 
[12] Möller, W., Borgesen, P., Scherzer, B. M. U., Nucl. Inst. and Methods B19/20 (1987) 826.

[13] Thomas, E. W., Braun, M., J. Nucl. Matl. 111-112 (1982) 654.

[14] Pontau, A. E., Baskes, M. I., Wilson, K. L., Haggmark, L. G., Bohdansky, J., et al, J. Nucl. Matl. 111-112 (1982) 651.

[15] Sone, K., McCracken, G. M., J. Nucl. Matl. 111-112 (1982) 606.

[16] Powell, G. L. Inert Gas Permeability of High Temperature Gas-Cooled Reactor

Coated Fuel Particles. ORNL, Report Y/DA-7885, 1978.

[17] Halsey, G. D. in Trans. 8th Nat. Vac. Symp. 1, 119 (1962).

[18] Kornelsen, E. V., Can. J. Phys. 42 (1964) 364.

\section{Figure and Table Captions}

Figure 1 - When He is puffed into a low mode plasma (solid curve), with its unconditioned limiter, the He recycles until the end of the beam pulse at $t=1.0 \mathrm{~s}$. When the limiter has been depleted of D and TFTR is making supershots, the puff is pumped out during the shot by the limiter (dashed curve). (The time scale starts when the beam turns on. The He was puffed at $0.45 \mathrm{~s}$ for the low mode and $0.40 \mathrm{~s}$ for the supershot.)

Figure 2 - This is a series of neon injections into TFTR while the limiter is being conditioned. The same amount of $\mathrm{Ne}$ was injected into each shot. The 3 shots shown are separated by 7 conditioning shots each. As the machine becomes more conditioned, the pre-injection Zeff rises due to the decreasing amount of deuterium available for recycling from the limiter. At a Zeff of 4 , the plasma is $25 \%$ carbon ions, which are sustained largely by self sputtering. As the deuterium inventory in the limiter is reduced, the neon pumps out faster and more completely.

Figure 3 - This is the line-integrated density signal from the third (highest $\mathrm{Z}_{\text {eff }}$ ) Ne injection shot shown in Fig. 2. At this point in the conditioning, the pumpout is quite well defined.

Figure 4 - This graph shows the brightness of the Ar XVI line at $354 \AA$ for three consecutive shots with increasing levels of argon $(0.23,1.47$, and $2.35 \mathrm{~T}-\ell$, from bottom to top) puffed during beam injection. The limiter is fully conditioned for these shots. Note that there is considerable pumpout of Ar between shots, although there is clear evidence of a buildup, seen in the ohmic phase levels before neutral beam or Ar injection. The low-level NBI from 4.0 to 5.0 seconds while the plasma cools and the Ar pumps out is to prevent radiative collapse of the plasma and a possible disruption.

Figure 5 - After the Ar puffing run of in Fig. 4, the limiter outgassed Ar overnight. Here is the partial pressure peak of ${ }^{40} \mathrm{Ar}$ from the TFTR residual gas analyzer. The fitted exponential on a pedestal gives a time constant of 2.14 hours. The residuals of that fit, in percent deviation, are shown below. A single exponential is clearly not the form of the real behavior.

Figure 6 - This is the ${ }^{40} \mathrm{Ar}$ outgassing data shown in Fig. 5, but here fitted with two 
exponentials. Notice that the residuals show no systematic trends for this fit. Compare the proportions of the Ar outgassing with the short time constant and the long time constant with the Ne data shown in Table 2. The two gases seem to be sharing the same trapping sites, although the trapping depth is smaller for the lighter species.

Figure 7 - After we had run an Ar glow discharge in TFTR but had not made any highpower plasmas, we examined the ${ }^{40} \mathrm{Ar}$ outgassing peak. Compare the residuals with the $\mathrm{Ar}$ outgassing data in Figs. 5 and 6 above. In this case, there is no short time constant. All the outgassing is governed by a single long time constant.

Table 1 - Here is a summary of the pumping times for deuterium and the noble gases in THTR with a well conditioned graphite limiter. These are times for a 1.4 MA plasma current. There is little difference between effective confinement times for the noble gases in ohmic and beam heated plasmas.

Table 2 - This is a summary of the limiter outgassing after operations with noble gases. By "run" we mean normal high power plasma operation, usually with neutral beam heating. The limiter outgassing data for D after the Ar glow and subsequent run is shown for comparison with the Ar. Although the D outgassing is clearly a 2 exponential decay, and the time constants are comparable with those for Ar, the fraction in the long and the short components is quite different from that for the $\mathrm{Ar}$, and suggests that $\mathrm{D}$ is not using the same trapping sites as Ar. 


\begin{tabular}{|c|c|}
\hline Gas & $\tau_{\mathrm{p}}^{*}$, \\
\hline $\mathrm{D}$ & 0.12 \\
\hline $\mathrm{He}$ & 1.00 \\
\hline $\mathrm{Ne}$ & 0.70 \\
\hline $\mathrm{Ar}$ & 0.20 \\
\hline $\mathrm{Kr}$ & 0.08 \\
\hline
\end{tabular}

Table 1 - Here is a summary of the pumping times for deuterium and the noble gases in TFTR with a well conditioned graphite limiter. These are times for a 1.4 MA plasma current. There is little difference between effective confinement times for the noble gases in ohmic and beam heated plasmas. 


\begin{tabular}{|c|c|c|c|c|}
\hline Data Set & $\tau_{\mathrm{p}}$ (short), hrs & Fraction(short) & $\tau_{\mathrm{p}}($ long), hrs & Fraction(long) \\
\hline $\mathrm{Ne}$ (run) & $0.30 \pm 0.03$ & $31 \pm 3 \%$ & $1.8 \pm 0.1$ & $69 \pm 3 \%$ \\
\hline Ar (run) & $0.87 \pm 0.05$ & $34 \pm 3 \%$ & $4.1 \pm 0.3$ & $66 \pm 3 \%$ \\
\hline Ar (Ar glow) & $(0.2 \pm 1.0)$ & $(1 \pm 10 \%)$ & $5.3 \pm 0.1$ & $99 \pm 1 \%$ \\
\hline Ar (Ar glow+run) & $1.0 \pm 0.4$ & $65 \pm 7 \%$ & $4.9 \pm 0.7$ & $34 \pm 5 \%$ \\
\hline D (Ar glow+run) & $0.49 \pm 0.02$ & $17 \pm 2 \%$ & $4.2 \pm 0.1$ & $83 \pm 2 \%$ \\
\hline
\end{tabular}

Table 2 - This is a summary of the limiter outgassing after operations with noble gases. By "run" we mean normal high power plasma operation, usually with neutral beam heating. The limiter outgassing data for $D$ after the Ar glow and subsequent run is shown for comparison with the Ar. Although the $D$ outgassing is clearly a 2 exponential decay, and the time constants are comparable with those for Ar, the fraction in the long and the short components is quite different from that for the Ar. 


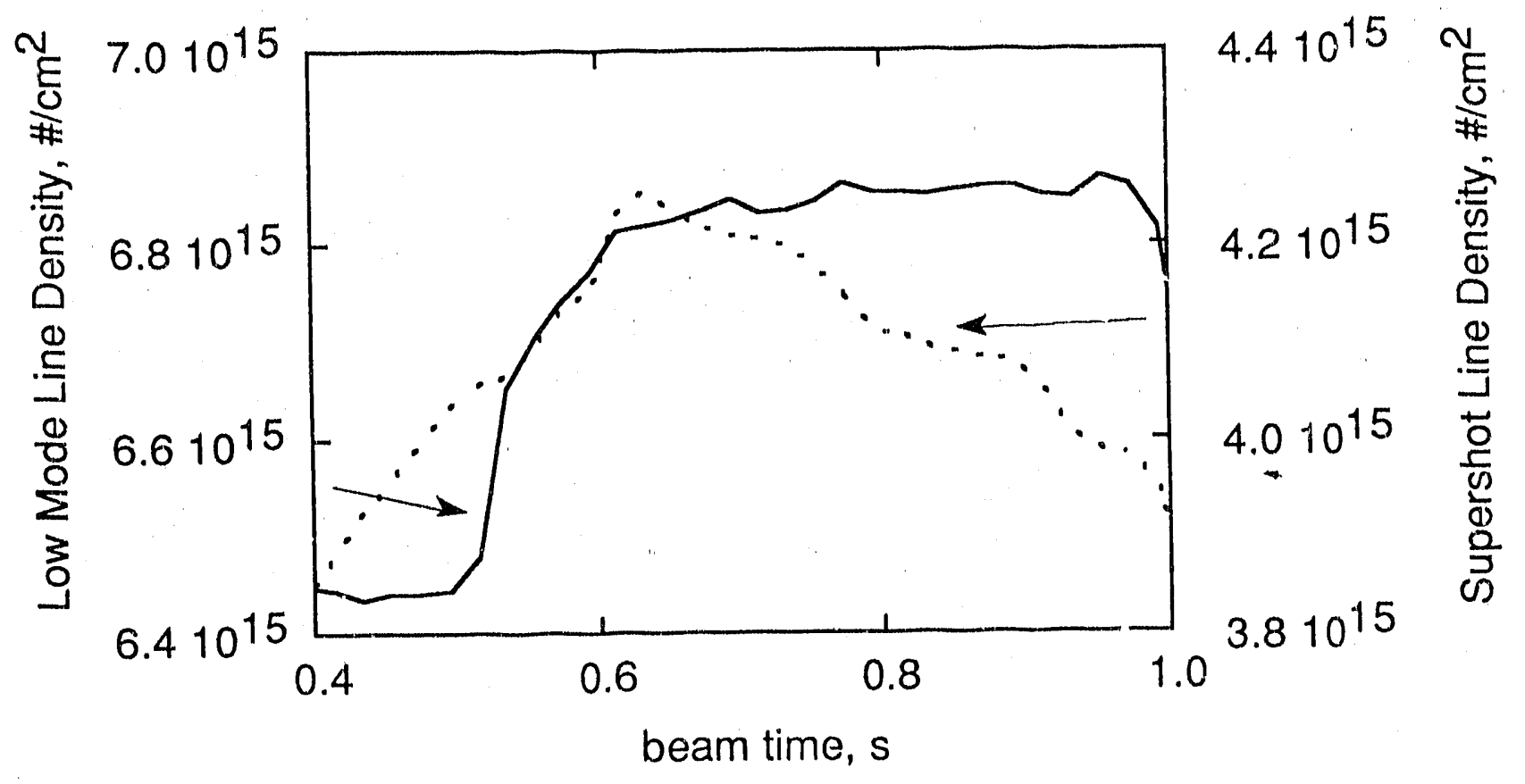

Figure 1 - When He is puffed into a low mode plasma (solid curve), with its unconditioned limiter, the $\mathrm{He}$ recycles until the end of the beam pulse at $t=1.0 \mathrm{~s}$. When the limiter has been depleted of $D$ and TFTR is making supershots, the puff is pumped out during the shot by the limiter (dashed curve). (The time scale starts when the beam turns on. The He was puffed at $0.45 \mathrm{~s}$ for the low mode and $0.40 \mathrm{~s}$ for the supershot.) 


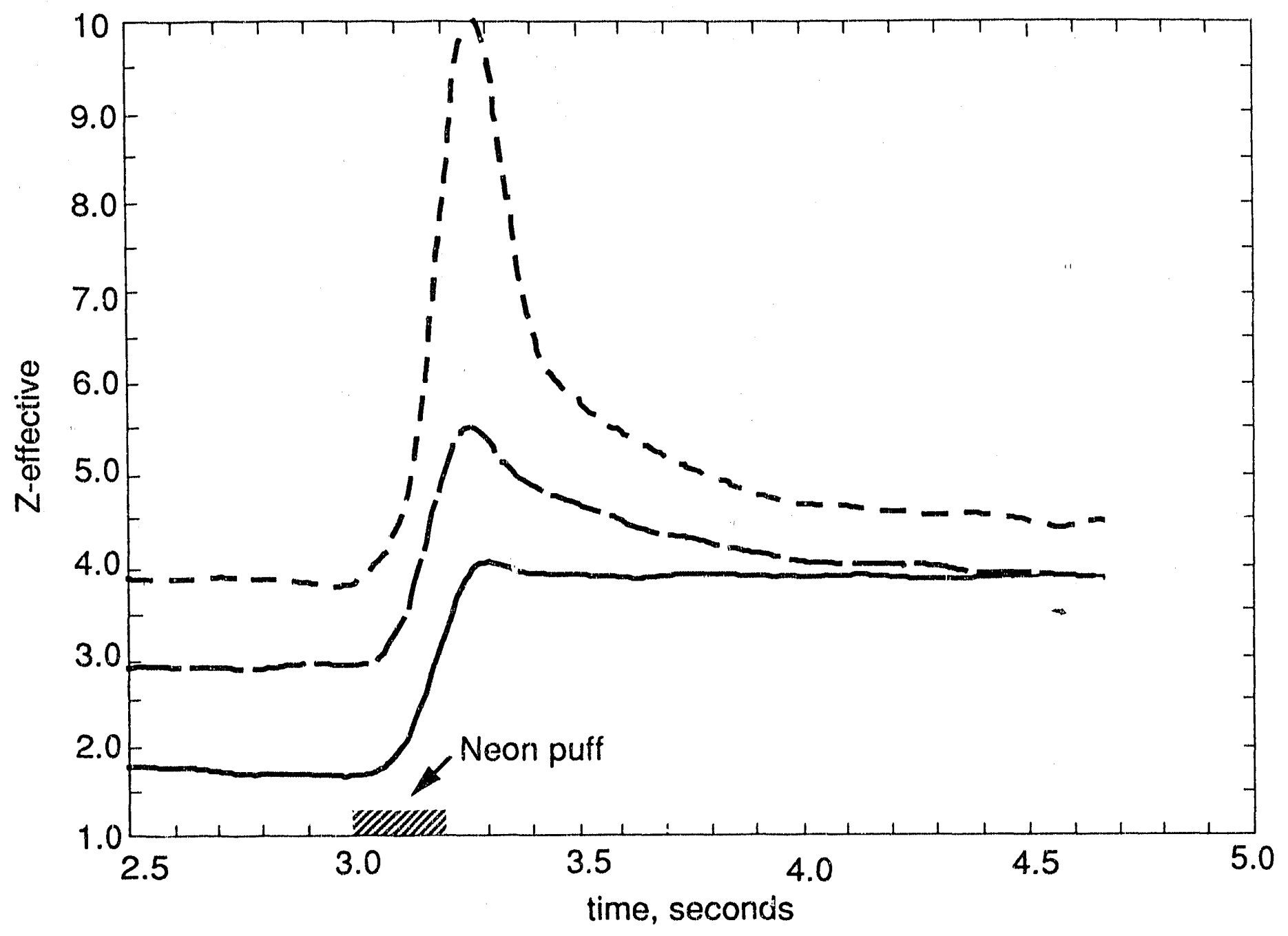

Figure 2 - This is a series of neon injections into TFTR while the limiter is being conditioned. The same amount of $\mathrm{Ne}$ was injected into each shot. The 3 shots shown are separated by 7 conditioning shots each. As the machine becomes more conditioned, the pre-injection $\mathrm{Z}_{\text {eff }}$ rises due to the decreasing amount of deuterium available for recycling from the limiter. At $a Z_{\text {eff }}$ of 4 , the plasma is $25 \%$ carbon ions, which are sustained largely by self sputtering. As the deuterium inventory in the limiter is reduced, the neon pumps out faster and more completely. 


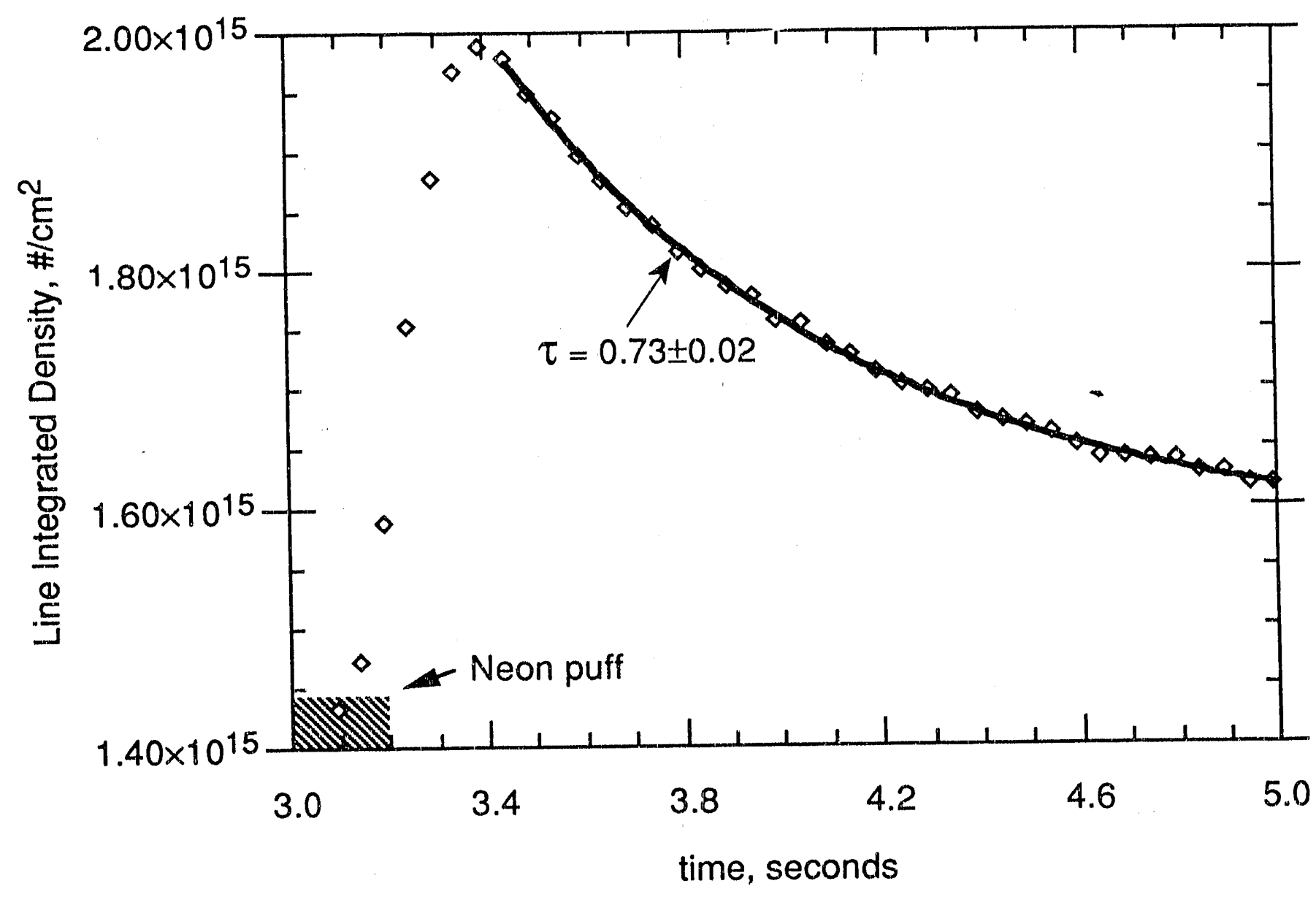

Figure 3 - This is the line-integrated density signal from the third (highest $\left.\mathrm{Z}_{\text {eff }}\right) \mathrm{Ne}$ injection shot shown in Fig. 2 . At this point in the conditioning, the pumpout is quite well defined. 


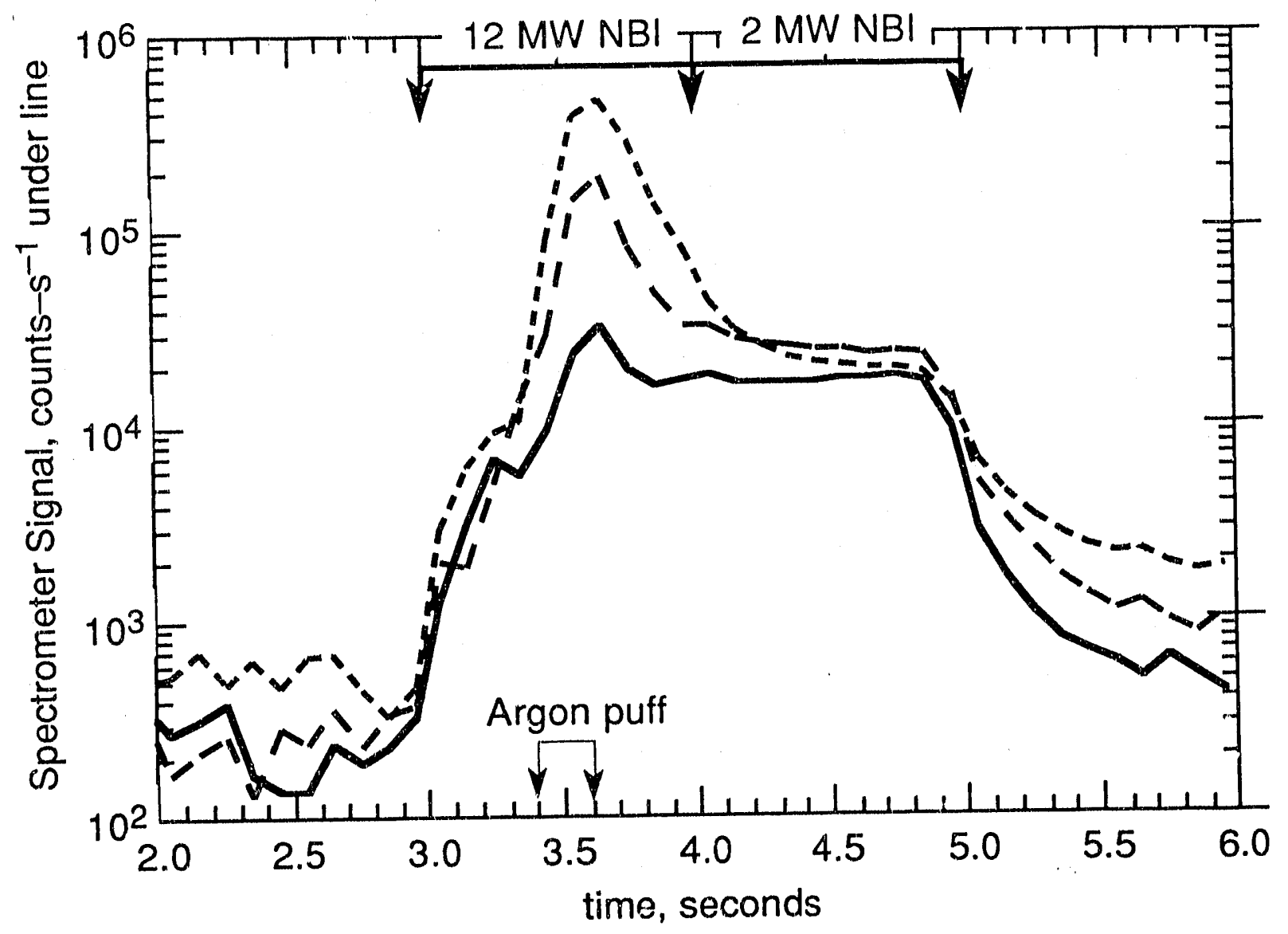

Figure 4 - This graph shows the brightness of the Ar XVI line at $354 \AA$ for three consecutive shots with increasing levels of argon $(0.23,1.47$, and $2.35 \mathrm{~T}-\ell$, from bottom to top) puffed during beam injection. The limiter is fully conditioned for these shots. Note that there is considerable pumpout of Ar between shots, although there is clear evidence of a buildup, seen in the ohmic phase levels before neutral beam or Ar injection. The low-level NBI from 4.0 to 5.0 seconds while the plasma cools and the Ar pumps out is to prevent radiative collapse of the plasma and a possible disruption. 

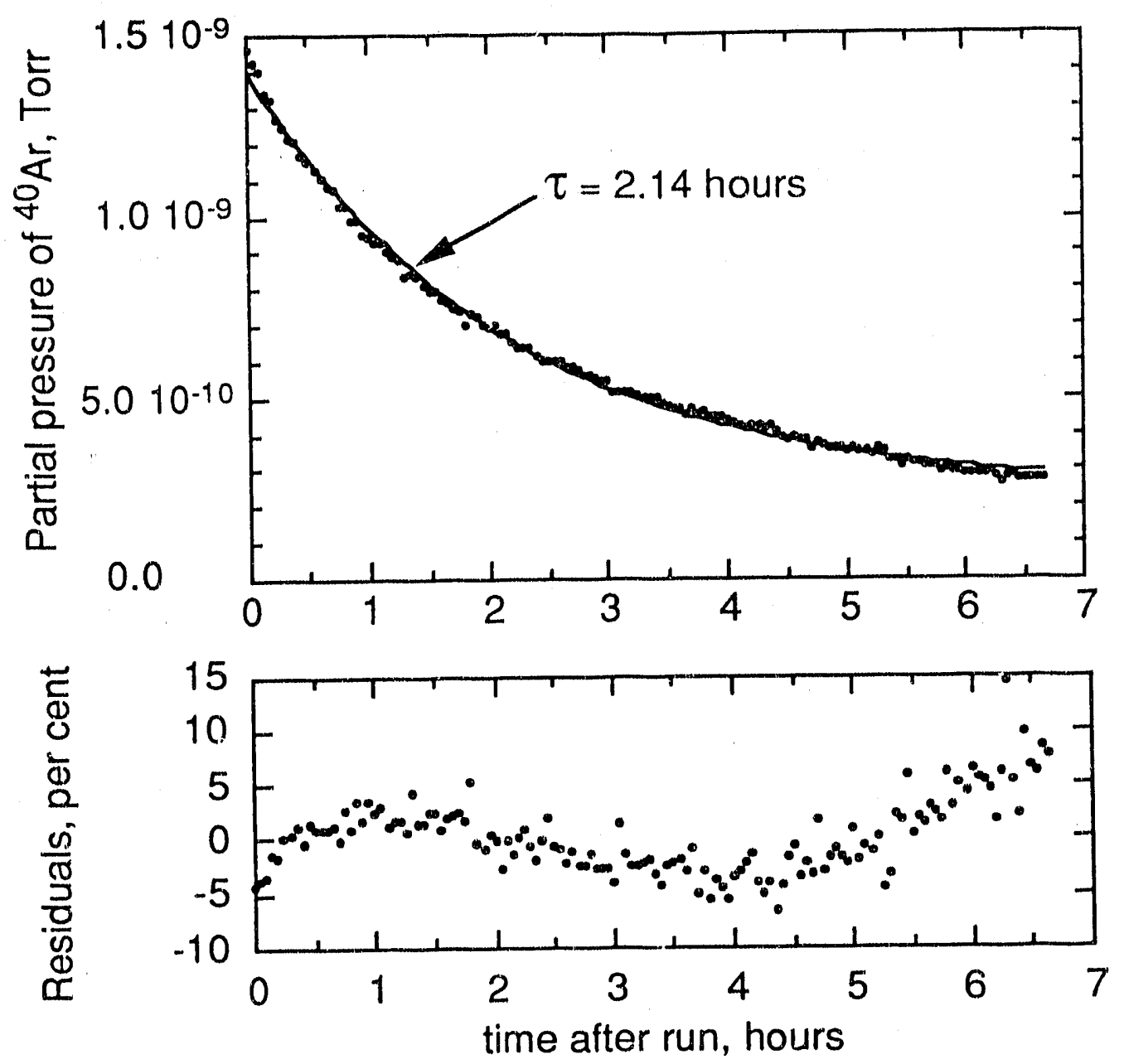

Figure 5 - After the Ar puffing run of in Fig. 4, the limiter outgassed $\mathrm{Ar}$ overnight. Here is the partial pressure peak of ${ }^{40} \mathrm{Ar}$ from the TFTR residual gas analyzer. The fitted exponential on a pedestal gives a time constant of 2.14 hours. The residuals of that fit, in percent deviation, are shown below. A single exponential is clearly not the form of the real behavior. 

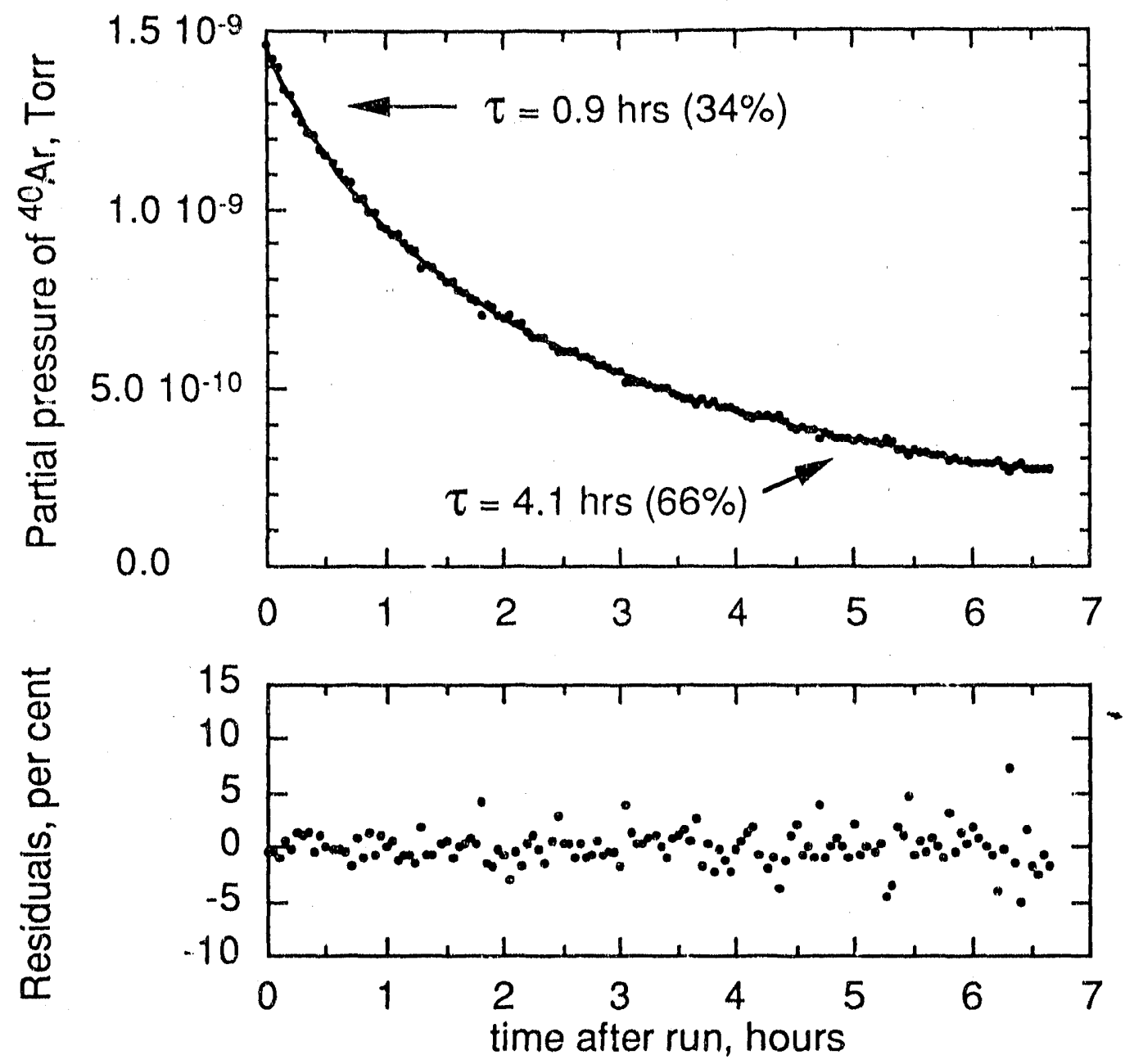

Figure 6 - This is the ${ }^{40} \mathrm{Ar}$ outgassing data shown in Fig. 5, but here fitted with two exponentials. Notice that the residuals show no systematic trends for this fit. Compare the proportions of the Ar outgassing with the short time constant and the long time constant with the Ne data shown in Table 2. The two gasses seem to be sharing the same trapping sites, although the trapping depth is smaller for the lighter species. 

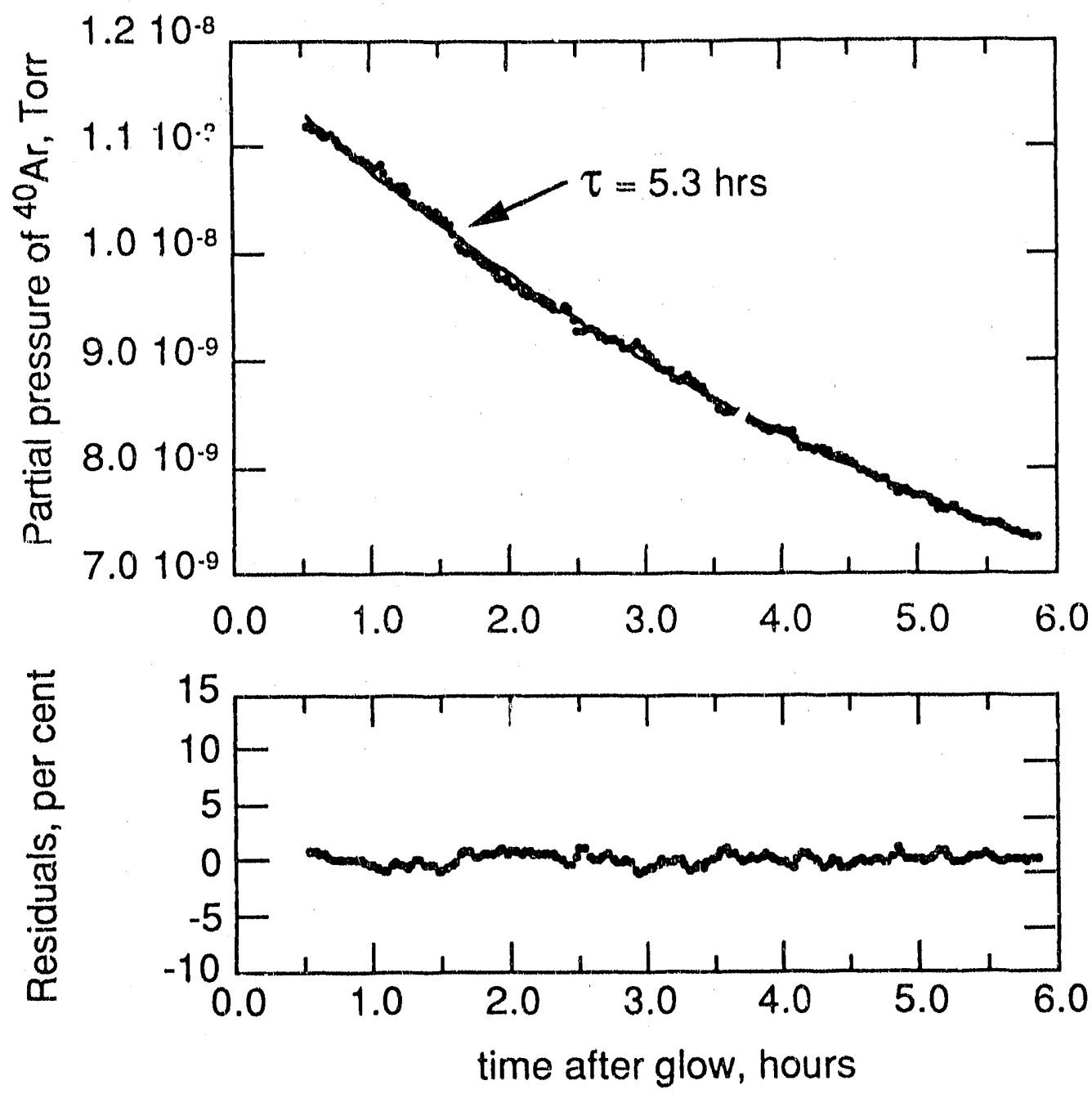

Figure 7 - After we had run an Ar glow discharge in TFTR but had not made any high-power plasmas, we examined the ${ }^{40} \mathrm{Ar}$ outgassing peak. Compare the residuals with the Ar outgassing data in Figs. 5 and 6 above. In this case, there is no short time constant. All the outgassing is governed by a single long time constant. 

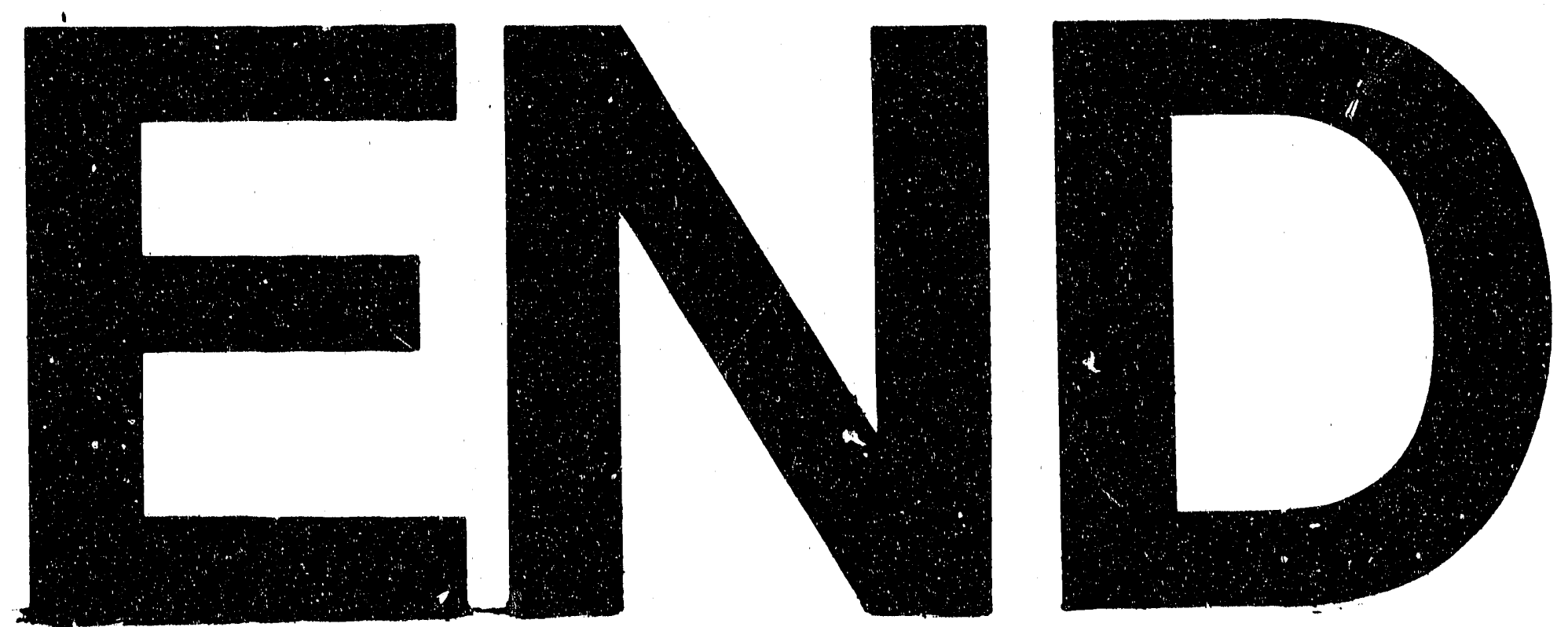

- 1
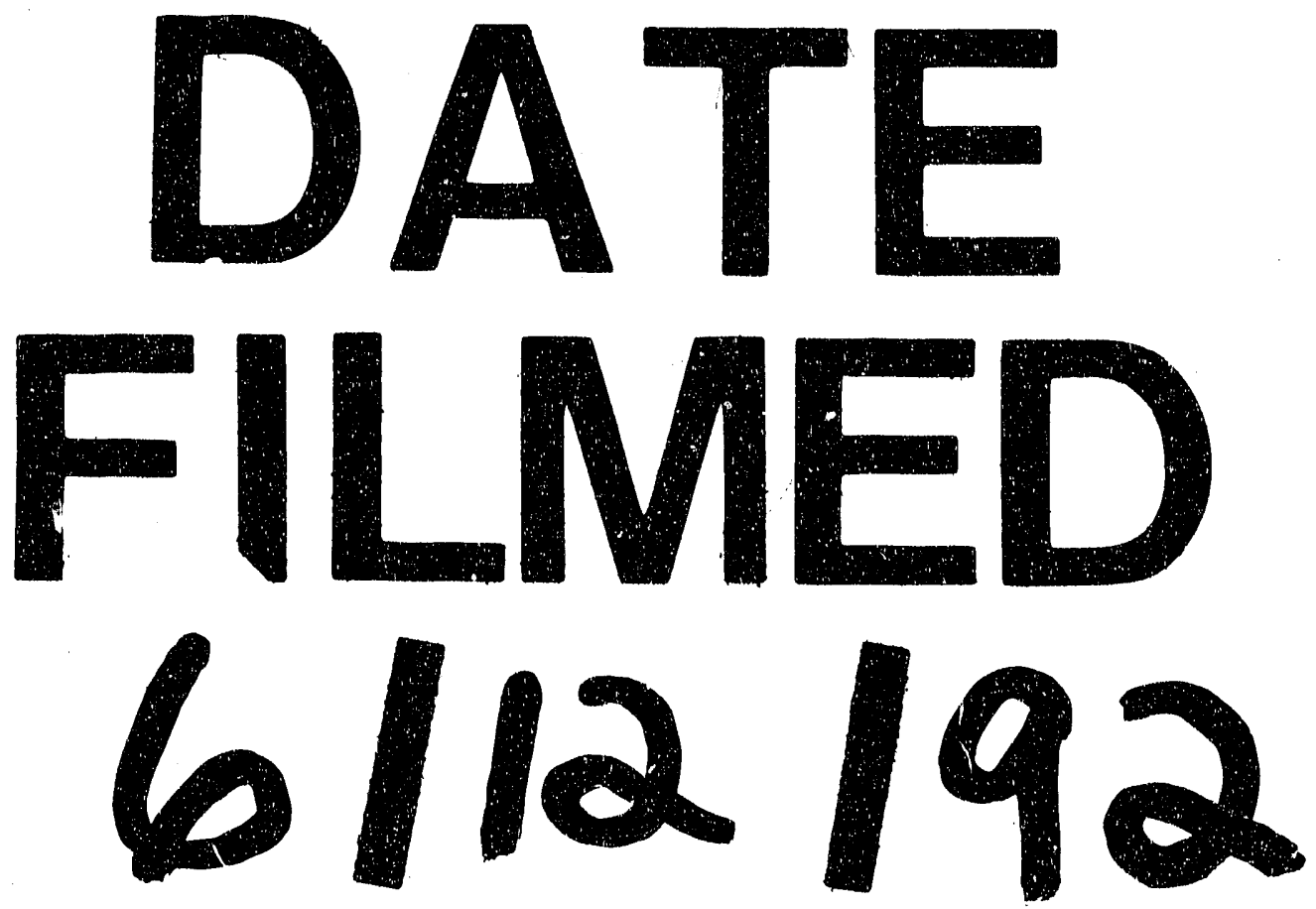
